\title{
Left Sided Mc Connells Sign: A Classic Finding in a Rare Condition
}

\author{
Eric McWilliams, Ehab Amer and Ali Alshehri \\ Cardiology Department, Johns Hopkins Aramco Healthcare, Dhahran, Saudi Arabia \\ eric.mcwilliams@gmail.com; amerehab@hotmail.com; ehab.amer@jhah.com
}

\begin{abstract}
A 32 year old lady with a history of systemic lupus erythematosus (SLE) complicated by crescentic lupus nephritis and end stage renal failure on thrice weekly haemodialysis, presented to the emergency department with breathlessness, chest pain and haemoptysis, stating that her symptoms had started roughly 24 hours earlier. She appeared anxious and in distress. BP was 160/105 mmHg, heart rate $105 /$ min and there was evidence of bilateral rales and a gallop rhythm. She was afebrile but only $94 \%$ saturating on high flow oxygen.
\end{abstract}

ECG showed left ventricular hypertrophy and non-specific changes, chest X-ray demonstrated bilateral patchy infiltrates and cardiomegaly(figure 1). White cell count was $13.7 \mathrm{~K}, \mathrm{CRP} 2.1$, Creatinine 8.6, BUN $46, \mathrm{~K} 4.6$ and high sensitivity troponin elevated at 8.755 ( $\mathrm{ref}<0.03$ ).

Echocardiography (Video 1) showed severe hypokinesis of the septum, anterior wall and basal inferior wall with an estimated ejection fraction of $40 \%$ but preserved or even hyperkinetic apical function. (figure $1 \&$ Video 2) There was evidence of high filling pressures and pulmonary hypertension. (figure 2) Speckle tracking echo (STE) demonstrated preserved apical strain (figure 2) with markedly reduced strain in the basal segments.

A presumptive diagnosis of basal variant Taku tsubo cardiomyopathy was made and the patient admitted to the intensive care unit. She was treated by emergent haemodialysis and was commenced on betablockade in the form of Carvedilol. She demonstrated rapid clinical improvement. She declined invasive workup with coronary angiography. There were no significant serial EKG changes.

Repeat echocardiography (Video 3\&4)was performed 48 after presentation and demonstrated normalisation of LV systolic function as well marked improvement in diastolic parameters ( Figure 3 ).Speckle tracking echo also showed marked improvement in comparison with the echo on presentation(Figure 4). At this stage, the patient insisted on discharge and she has remained well at follow up. In retrospect, she admitted that she had become distraught with a work colleague during an argument immediately before her symptoms commenced. The rapid recovery of LV function and the non-coronary distribution of LV dysfunction excludes other aetiologies such as coronary embolism, plaque rupture or dissection.

The echocardiogram shows classic basal type taku tsubo cardiomyopathy(TTC), with preserved apical function. Four distinct variants of TTC have been described. Basal type TTC is rare and reported in 2.2\% of TTC cases (39 of 1750 cases) in the international TTC registry ${ }^{1}$. Apical type is the commonest $81.7 \%$; 
Eric McWilliams, Ehab Amer and Ali Alshehri; Left Sided Mc Connells Sign: A Classic Finding in a Rare Condition. Journal of Biomedical Engineering and Medical Imaging, Volume 3, No 5, October (2016), pp 14-17

midventricular type occurs in $14.6 \%$ and focal is rare occurring in only $1.5 \%^{1}$. TTC can occur in younger females but the risk is $4.8 \%$ greater in females $>55$ years. The traditional wisdom is that TTC is often precipitated by emotional stress but physical factors actually predominated as the commonest precipitating factor in the international registry. ${ }^{1}$

The diagnosis of TTC is usually confirmed by the exclusion of significant obstructive coronary artery disease by invasive angiography, particularly in patients presenting with a syndrome suggesting ACS. However, it may not always be possible to perform coronary angiography and echocardiography plays a pivotal role in the diagnosis, risk stratification and follow up of TTC patients. ${ }^{2}$ Advanced echocardiographic techniques such as STE, myocardial contrast and coronary flow studies may be incorporated into an algorithm than can obviate the need for invasive angiography in some patients. ${ }^{2}$

McConnell's sign is a specific finding in cases of acute pulmonary embolism. Mc Connells sign ${ }^{3}$ is defined as right ventricular free wall akinesis with sparing of RV apical contraction and is considered a specific echocardiographic finding in acute pulmonary embolism.

The presence of hyperkinetic motion of the basal and mid segments of the RV free wall with RV apical akinesis suggests right ventricular involvement in TTC and this finding has been suggested as "reverse McConnell's sign". ${ }^{4}$ LV apical hyperkinesis with basal and midventricular hypokinesis could be considered a "left sided Mc Connell's sign" and should suggest basal or midventricular variants of TTC.

Disclosures:

None of the authors have any disclosures.

\section{REFERENCES}

[1] Clinical Features and outcomes of Taku Tsubo ( stress ) Cardiomyopathy:n engl j med 373;10 nejm.org September 3, 2015

[2] Standard and Advanced Echocardiography in Taku Tsubo ( Stress ) Cardiomyopathy: Clinical and Prognostic Implications. j.echo.2014.08.020

[3] Regional right ventricular dysfunction detected by echocardiography in acute pulmonary embolism. McConnell MV, Solomon SD, Rayan ME, Come PC, Goldhaber SZ, Lee RT. Am J Cardiol. 1996 Aug 15;78(4):469-73.

[4] "Reverse McConnell's Sign?": A Unique Right Ventricular Feature of Takotsubo Cardiomyopathy: Kan Liu, MD, PhD Robert Carhart, MD. AM J Cardiol.2013 April 15; 111, Issue 8: 1232-1235 


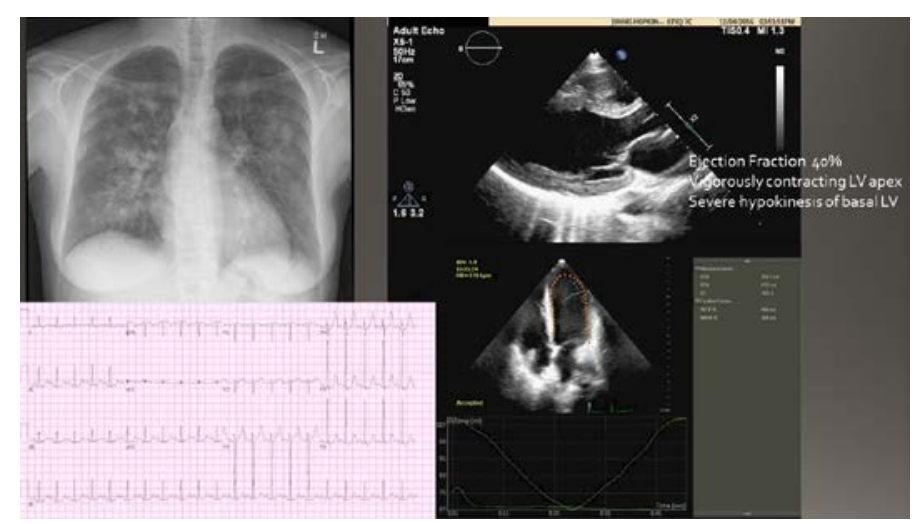

Figure 1: Panel A: Chest X-ray showing pulmonary edema. Panel B: EKG. Panel C: Transthoracic echo PLAX view at presentation. Panel D: initial echo apical view

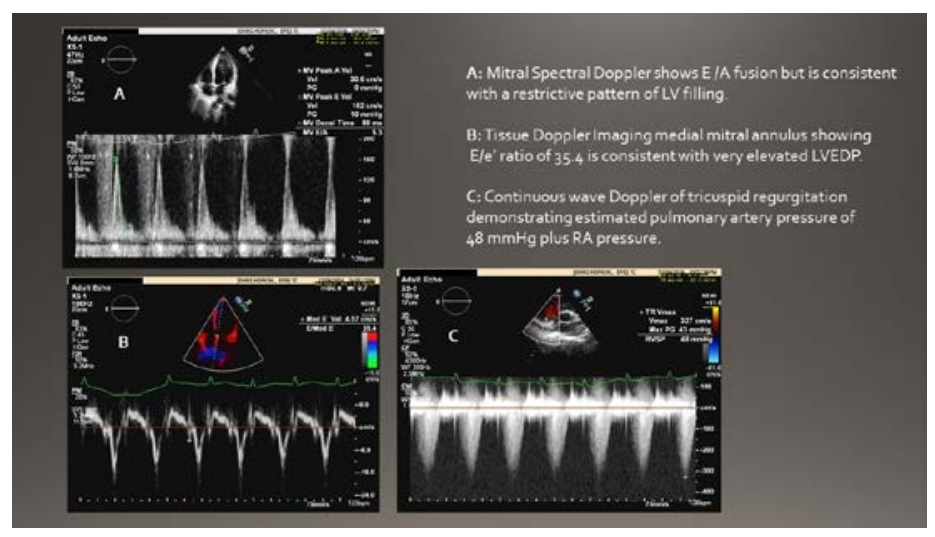

Figure 2: Echo Doppler findings at presentation. Panel A: pulsed Doppler mitral inflow. Panel B: tissue Doppler septal annulus. Panel C: continuous wave Doppler tricuspid valve

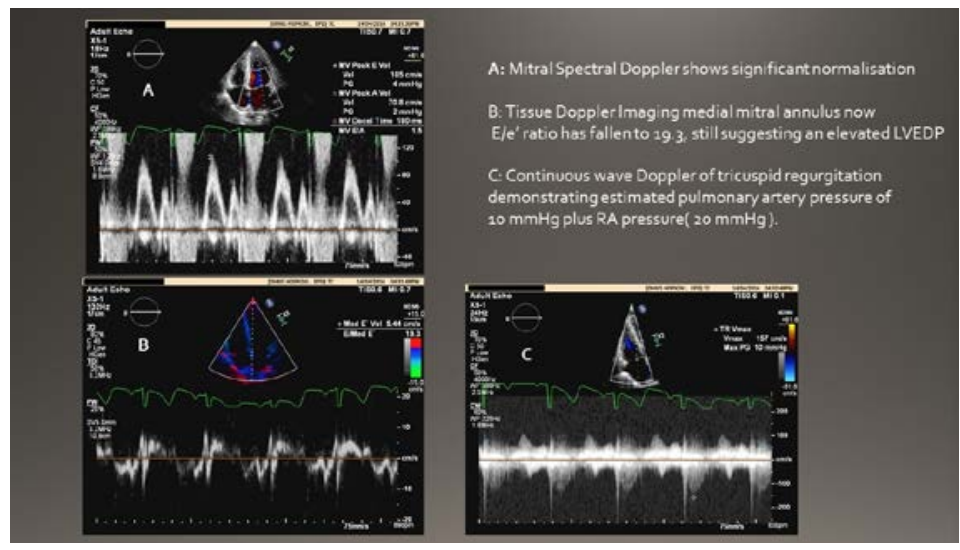

Figure 3: Echo Doppler findings at 48 hours. Panel A: pulsed Doppler mitral inflow. Panel B: tissue Doppler septal annulus. Panel C: continuous wave Doppler tricuspid valve 


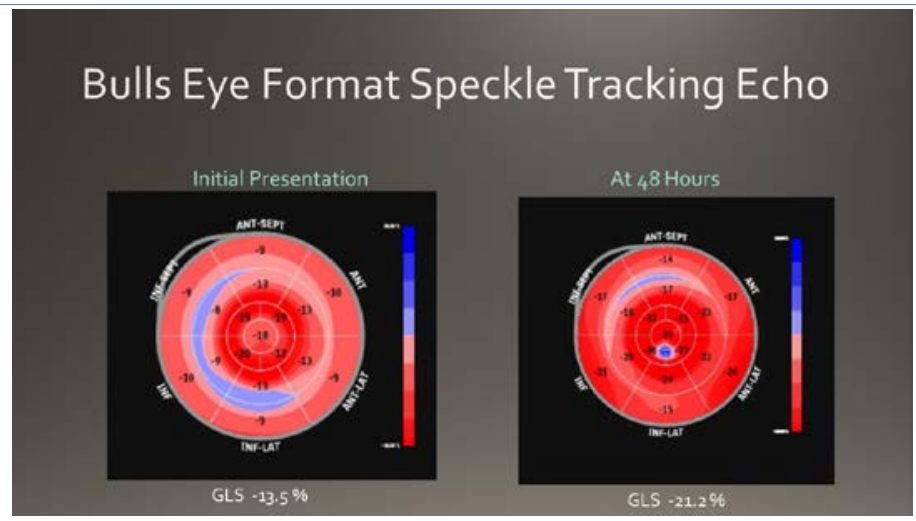

Figure 4: Speckle tracking echo in bulls eye format demonstrating longitudinal strain. Left panel at presentation. Right panel after $\mathbf{4 8}$ hours.

\section{VIDEOS}

Video 1: Transthoracic echo, parasternal long axis view at presentation http://scholarpublishing.org/Repository/JBEMi/Video1.wmv

Video 2: Transthoracic echo, apical 4 chamber view at presentation http://scholarpublishing.org/Repository/JBEMi/Video2.wmv

Video 3: Transthoracic echo, parasternal long axis view at 48 hours http://scholarpublishing.org/Repository/JBEMi/Video3.wmv

Video 4: Transthoracic echo, apical 4 chamber view at 48 hours http://scholarpublishing.org/Repository/JBEMi/Video4.wmv 